\title{
Boron Diffusion in Strained and Strain-Relaxed SiGe
}

\author{
C.C. Wang, T.Y. Huang, Y. M. Sheu, Ray Duffy ${ }^{1}$, Anco Heringa ${ }^{1}$, \\ N.E.B. Cowern ${ }^{2}$, Peter B. Griffin ${ }^{3}$, Carlos H. Diaz* \\ TCAD project, Device Engineering Division, R\&D, \\ *Advanced Device Engineering Department, Logic Technology Division, R\&D \\ Taiwan Semiconductor Manufacturing Company, \\ Hsin-Chu, Taiwan, 300-77, R.O.C. (ccwangd@tsmc.com) \\ ${ }^{1}$ Philips Research Leuven, Kapeldreef 75, 3001 Leuven, Belgium \\ ${ }^{2}$ Advanced Technology Institute, University of Surrey, Surrey GU2 7XH, UK \\ ${ }^{3}$ Center for Integrated Systems, Stanford University, Stanford, CA, 94305-4070, USA
}

\begin{abstract}
SiGe has been utilized for aggressive CMOS technologies development recently and there are many literatures talking about the advantages brought by it. However, few publications discuss the impacts from both mechanical strain and Ge doping on boron diffusion. Moreover these effects have mostly been studied at low boron concentrations and with long high temperature anneals. They are not the possible conditions used in aggressive CMOS technologies. An experiment has been therefore designed to investigate boron diffusion in both strained and strain-relaxed SiGe including ultra-low energy, high concentration boron implant and spike RTA. Summarily, this paper describes the experiments, calibration and resulting diffusion constants for an ultra-shallow boron junction in $\mathrm{SiGe}$ that is popular in advanced CMOS technology.
\end{abstract}

\section{Introduction}

The hole mobility enhancement due to strain from various SiGe layer growth approaches has been widely adopted for aggressive CMOS technologies[1][2]. In addition to this advantage, the retarded diffusion of boron in $\mathrm{SiGe}$ is also a benefit for device scaling. Boron diffusivity under this circumstance has been modeled in $[3,4]$ with a diffusivity retardation that is an exponential function of the strain. Alternatively, it is claimed that the retardation is from Ge-B pairing instead [5,6]. A quantitative assessment of the respective contribution from mechanical strain and $\mathrm{Ge}$ doping is therefore necessary for accurate boron modeling in SiGe. This paper will investigate boron diffusion in both strained and strain-relaxed SiGe including ultralow energy, high concentration boron implant and spike RTA. The effects of implant damage, boron-interstitial cluster (BIC) model and TED effects will be also considered at the modeling procedure.

\section{Experiments}

There were two experiments performed in this work. One (experiment A) is a relaxed SiGe experiment, and another (experiment B) is a strained SiGe experiment. A 2- $\mu \mathrm{m}$ thick relaxed $\mathrm{SiGe}$ layer was deposited as described in [7], with various $\mathrm{Ge}$ concentrations, $9 \%, 15 \%$, and $28 \%$ for experiment A. Wafers in experiment B were implanted with Ge at a dose of $1 \times 10^{16} \mathrm{~cm}^{-2}$ at a variety of energies $5 \mathrm{keV}, 10 \mathrm{keV}$, $15 \mathrm{keV}$ and $20 \mathrm{keV}$. Following the Ge implant, the wafers were annealed in an inert $\mathrm{N}_{2}$ ambient to form a strained SiGe layer. A Boron implant followed with a dose of $1.3 \times 10^{15} \mathrm{~cm}^{-2}$ and an energy of $2 \mathrm{keV}$ for both experiments A and B. Finally, all wafers 
were annealed by a high temperature spike RTA that is widely used for ultra-shallow junction (USJ) formation. Dopant profiles were analyzed by secondary ion mass spectrometry (SIMS). Table 1 shows the process flow and detailed conditions. Once the process was completed, both B and Ge profiles were extracted with SIMS, using $750 \mathrm{eV} \mathrm{O} 2+$ primary ions with an oblique angle of incidence $\left(45^{\circ}\right)$ and $2 \times 10^{-6}$ torr oxygen-leak.

\begin{tabular}{|c|c|}
\hline Experiment A & Experiment B \\
\hline $2-\mu \mathrm{m}$ SiGe & Ge implant $1 \mathrm{E} 16 / \mathrm{cm}^{2}$ \\
film deposition & $5,10,20,30 \mathrm{keV}$ \\
\cline { 2 - 2 } $9 \%, 15 \%, 28 \%$ & SiGe film formation \\
& by furnace anneal \\
\hline Boron implant & Boron implant \\
$2 \mathrm{keV} / 1.3 \mathrm{E} 15$ & $2 \mathrm{keV} / 1.3 \mathrm{E} 15$ \\
\hline Spike RTA & Spike RTA \\
$970^{\circ} \mathrm{C}, 1070^{\circ} \mathrm{C}$ & $1050^{\circ} \mathrm{C}$ \\
\hline
\end{tabular}

Table 1 : Detailed process conditions at experiments A and B.

\section{Model Calibration and Discussion}

Figures 1 - 2 show the SIMS profiles for experiment A annealed at $970^{\circ} \mathrm{C}$ and $1070^{\circ} \mathrm{C}$ respectively. We fitted the retarded $\mathrm{B}$ diffusion in $\mathrm{SiGe}$ with a $\mathrm{Ge}$ concentration and anneal temperature dependent empirical equation that is shown below based on a temperature dependent Ge-B pairing model.

D_SiGe $=$ D_Si $x \operatorname{Exp}\left[-4.2 E-6 \times \operatorname{Exp}(1.58 / \mathrm{kT}) \times \mathrm{Ge}\right.$.Frac] $=\mathrm{D} \_S i \times \operatorname{Exp}(\Delta \operatorname{Ea})$ (1)

where D_Si is the diffusivity of Boron in silicon, T is the anneal temperature $(\mathrm{K})$ and Ge.Frac is germanium fraction. Contrary to the low boron concentrations used in $[6,8]$, a Fermi-level dependent diffusivity, fully-coupled model and boron-interstitial cluster (BIC) model are necessary to model experiments A and B. The boron diffusivity in relaxed $\mathrm{SiGe}$ is therefore well modeled at various anneal temperatures (Figs. 1 and 2). The retarded diffusivity versus Ge concentration is also shown in Fig. 3. Second, the calibrated empirical equations were modified to include a strain dependence of the Boron activation energy based on [3], and used in the calibration of experiment B (Fig. 4). The strain dependent change in the activation energy, $Q^{\prime}{ }_{\text {B(i) }}$ in [3], was extracted as $0.068 \mathrm{eV} /$ percent-strain, instead of $0.17 \mathrm{eV} /$ percent-strain found in [3]. The relationship between strain and diffusivity can be expressed as

D_SiGe $=$ D_Si x $\operatorname{Exp}(G e . s t r a i n \times(-6.8) / k T)=D \_S i x \operatorname{Exp}(\Delta E b)$

Our model is therefore expressed by including both $\mathrm{Ge}$ concentration $(\Delta \mathrm{Ea})$ and strain dependences $(\Delta \mathrm{Eb})$.

D_SiGe $=$ D_Si $x \operatorname{Exp}(\Delta \mathrm{Ea}+\Delta \mathrm{Eb})$

Figure 5 shows the detailed diffusivity retardation component under both mechanical strain and Ge doping. The retardation effect from mechanical strain still plays a significant role in strained SiGe. 


\section{Conclusion}

We have calibrated USJ boron retarded diffusion in both relaxed and strained SiGe. The retardation components from Ge doping and mechanical strain are also identified. Empirical equations are used to model the dependence on $\mathrm{Ge}$ concentration and mechanical strain respectively. Based on the calibration result, we conclude both mechanical strain and Ge concentration dependent retardation significantly impact boron diffusion behavior in strained SiGe.

\section{References}

[1]M. Shima et al, VLSI Tech. Dig. pp. 94-95,2002

[2]T. Ghani et al, IEDM pp.978-980, 2003

[3]N.E.B. Cowern et al, Physical Review Letters, pp.2585-2588, Vol. 72, No.16, April, 1994

[4]C.L. Liu et al, Materials Research Society Sym. Proceedings, Vol. 765, pp. D5.9.1, 2003

[5]R.F. Lever et al, Journal of Applied Physics, pp.1988-1994, Vol. 83, No. 4, February, 1998

[6]P. Kuo et al, Applied Physics Letters, pp. 580-582, Vol. 66, No. 5, January, 1995

[7]C.C. Lin et al, pp. $12-13$, SSDM, 2002

[8]N.R. Zangenberg et al, Journal of Applied Physics, pp.3883-3890, Vol. 94, No. 6, Sep., 2003

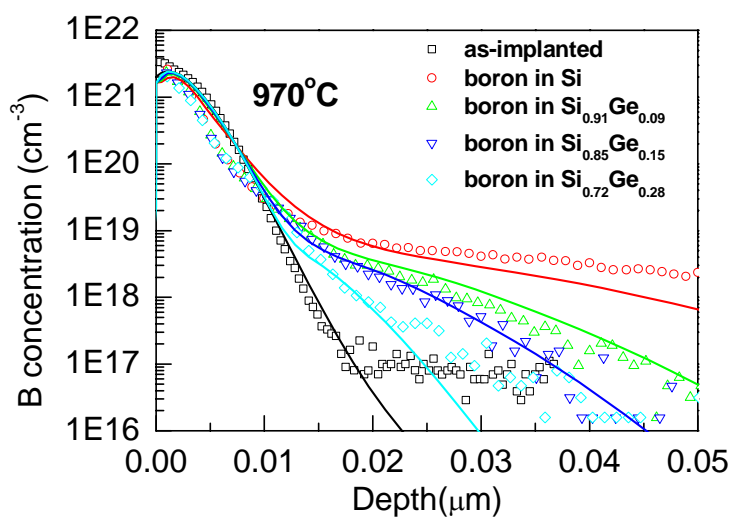

Figure 1 : Boron SIMS profiles (symbols) and calibration results (lines) based on equation 1 at experiment $\mathrm{A}$ annealed at $970^{\circ} \mathrm{C}$

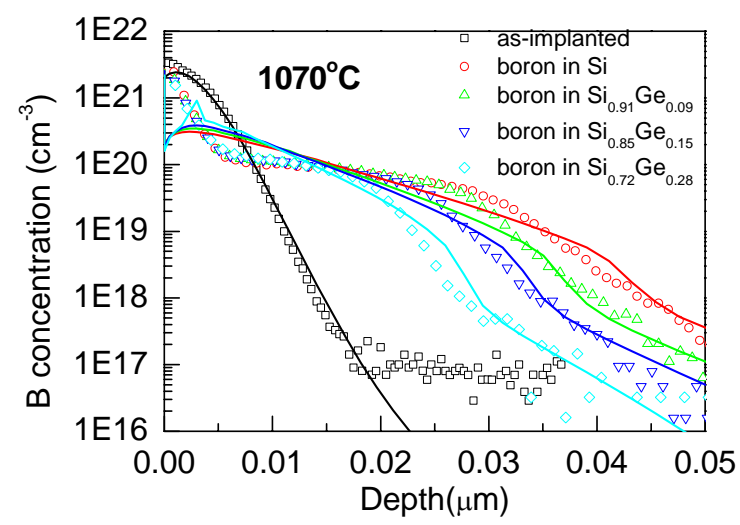

Figure 2 : Boron SIMS profiles (symbols) and calibration results (lines) based on equation 1 at experiment $\mathrm{A}$ annealed at $1070^{\circ} \mathrm{C}$. 


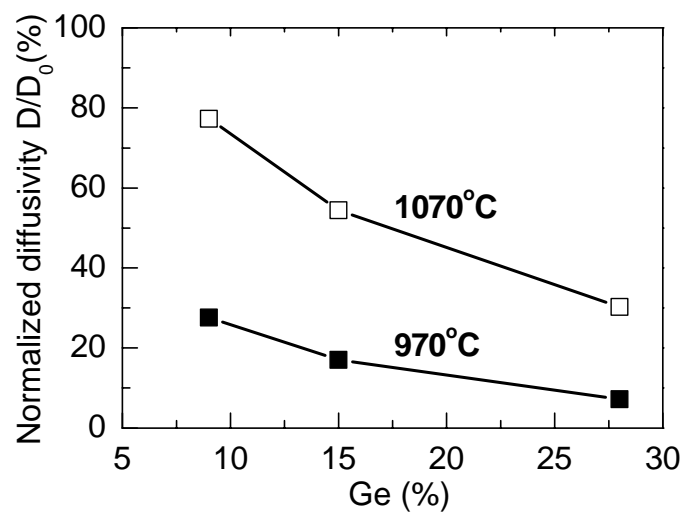

Figure 3 : The normalized diffusivity for a variety of Ge concentrations extracted from the calibration results shown in Figs. 1 - 2.

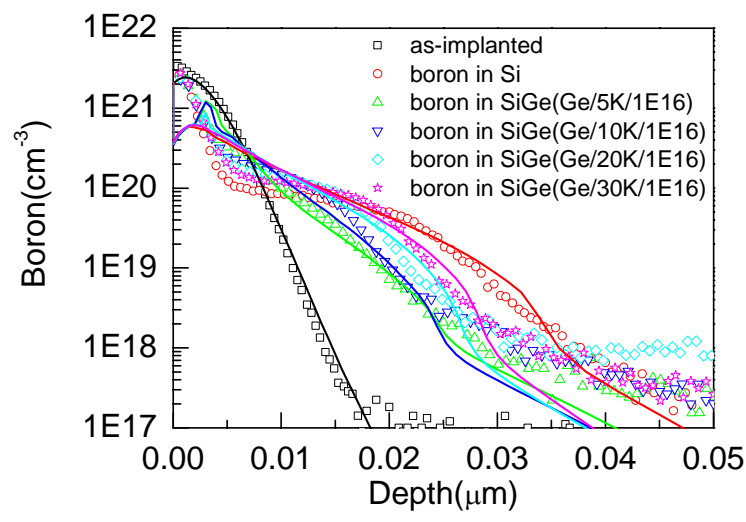

Figure 4 : SIMS profiles and calibration results based on both Ge-B pairing and strain effects at experiment B annealed at $1050^{\circ} \mathrm{C}$.

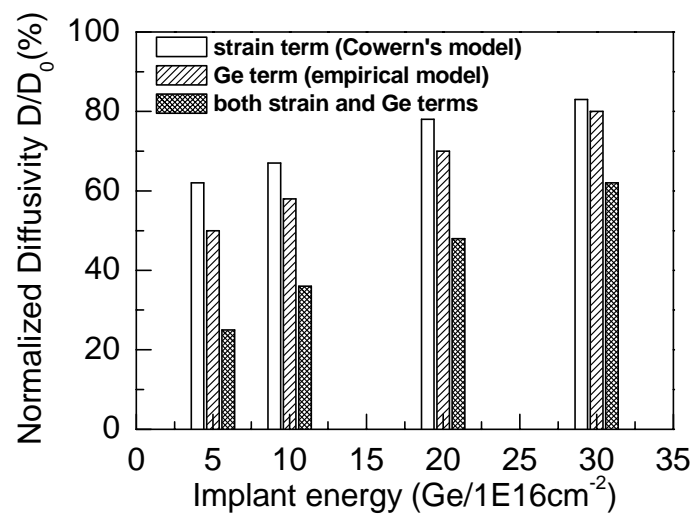

Figure 5 : The bar chart to show respective retardation contributions from various models at experiment $\mathrm{B}$. 